\title{
Social influences on eating: implications for nutritional interventions
}

\author{
Eric Robinson ${ }^{1}$, Jackie Blissett ${ }^{2}$ and Suzanne Higgs ${ }^{2 *}$ \\ ${ }^{1}$ Psychological Sciences, University of Liverpool, Liverpool L69 3GL, UK \\ ${ }^{2}$ School of Psychology, University of Birmingham, Birmingham B15 2TT, UK
}

\section{Abstract}

We review recent research on the effect of social context on food intake and food choice and assess the implications for nutritional interventions. We focus on studies of modelling of eating behaviour and the impact of perceived eating norms on the amounts and types of food that individuals eat. We suggest that social context influences eating via multiple mechanisms, including identity signalling and self-presentation concerns. However, building on existing theoretical models, we propose that social factors may be particularly influential on nutrition because following the behaviour of others is adaptive and social norms inform individuals about behaviours that are likely to be optimal ('if everyone else is doing it, I probably should be'). Guided by understanding of the potential underlying mechanisms, we discuss how social norms might be used to promote healthier nutrition.

Key words: Social norms: Social influence: Food intake: Food choice: Modelling

\section{Introduction}

The current 'obesogenic' food environment that is characterised by easy availability of 'junk' foods and large portion sizes poses a significant challenge for the promotion of healthy eating ${ }^{(1)}$. There is now good evidence that these 'external' environmental factors have influences on the diet that are associated with weight gain and poor health ${ }^{(1-3)}$. Another 'external' factor that has been reported to influence eating behaviour is social context. In a number of studies conducted during the 1990s, John De Castro and colleagues found that when individuals eat in social groups they eat substantially more than when eating alone. Moreover, the more people present, the more individuals tend to eat ${ }^{(4,5)}$. These striking findings have been replicated in a number of subsequent investigations and we now know some likely explanations of social facilitation effects. Social occasions result in longer meals and meals with friends are relaxing, prompting individuals to eat in a less inhibited manner than if they eat with strangers ${ }^{(6,7)}$.

More recently, a body of work has amassed that allows for examination of a more specific form of social influence: the perceived eating habits of others. Here we review this literature and discuss the potential reasons why other individuals' eating behaviours have powerful effects on food intake and choice. We also propose novel ways to promote healthier dietary behaviour based on these underlying mechanisms. We focus the review around two main areas of research: modelling of eating behaviour and the effects of perceived eating norms on food intake and choice.

\section{Modelling of food choices and intake}

Many studies have examined how the eating behaviour of fellow diners shapes what and how much we eat. This literature now spans over 30 years, with Herman et al. ${ }^{(8)}$ eloquently summarising the main findings up to 2003. Over the last decade new questions have been asked in the area and novel methodologies have been adopted. It is now possible to draw new conclusions about the mechanisms underlying modelling and the conditions under which modelling occurs.

\section{Early food intake modelling studies}

The first laboratory-based modelling studies tested whether pairing a participant with an actor who was instructed to eat a lot $v$. very little food (a 'confederate') influenced how much a 'real' participant ate. The confederate appeared to be another participant and the food tended to be a high-energy snack food. In these studies, when the confederate ate a lot the participant also ate a lot and when the confederate ate very little then so did the participant ${ }^{(9-12)}$. Some studies also included a control condition in which the participant ate alone, which enabled 
direct examination of whether the amount eaten by the confederate increases or decreases the amount of food eaten by the participants relative to what would be eaten normally. From this early literature both propositions are generally supported (see Conger et al. ${ }^{(9)}$, Goldman et $a l^{(12)}$ or Rosenthal \& $\left.\operatorname{Marx}^{(13)}\right)$, although the results are not so clear in some studies ${ }^{(14)}$.

A common feature of all these early studies was the use of a taste-test paradigm. The explanation given to participants for eating in the presence of someone else was that they were required to rate some foods together as part of a research study. The participant and confederate were left facing each other in a room with separate bowls of food and instructed to eat as much as they liked during the task. See Goldman et al. ${ }^{(12)}$ or Rosenthal \& $\operatorname{Marx}^{(13)}$ for detailed descriptions of this method. A methodological weakness is that these settings could be argued to be somewhat artificial. It is possible that because the participants were in a strange setting and unsure of how to complete the taste test, they may have been encouraged to follow the eating behaviour of the confederate.

\section{Recent food intake modelling studies}

Two more recent studies ${ }^{(15,16)}$ have replicated the modelling effect using the taste-test paradigm, but others have used more naturalistic settings, which may increase the validity of the findings. For example, in a study reported by Hermans et $a l^{(17)}$, participants ate together whilst watching television and were informed that snack food was provided to make the environment more natural. Similarly, the same researchers ${ }^{(18)}$ led participants to believe that their study was about the effect of breakfast on cognitive performance, so participants ate breakfast together in a naturalistic bar/restaurant setting ${ }^{(18)}$.

The effect of the presence of a high $v$. low intake confederate on snack food intake has been replicated many times, including two reports that participants in a high intake confederate condition ate twice as much as participants in a low intake condition (about $380 v .190 \mathrm{~kJ}$ (90 v. $45 \mathrm{kcal}$ ) of candy, respectively $)^{(17,19)}$. Feeney et al. ${ }^{(20)}$ conducted a study using a similar design and examined the effect of a low intake model $v$. no model on pizza snacking. When the confederate ate very little, food intake was $37 \%$ lower than when the confederate was absent (a reduction of approximately $630 \mathrm{~kJ}(150 \mathrm{kcal}))$. Hermans et al. ${ }^{(21)}$ also examined the modelling effect on intake of vegetable snacks and found that eating with a high intake model was associated with higher intake than eating with a low intake model.

Similar effects are observed for peer modelling in young people. Bevelander et al. ${ }^{(22)}$ examined the effect of a present model on the snack intake of normal-weight and overweight primary school children. The children completed a puzzle task concurrently with access to the snacks. Normal-weight and overweight participants modelled intake but the effect of a model eating a large amount of food was particularly pronounced in the overweight children. The modelling effect remained even when participants returned for a second session $2 \mathrm{~d}$ later and ate alone, which suggests that norm effects are long lasting.

A number of other recent snack food studies have assessed food intake modelling using dyad designs, whereby two 'real' participants are left alone to consume high-energy sweet snack food and the main analysis of interest is the correlation between dyad members' food intake. In line with the results from confederate studies, the intake of one member of the dyad is closely rated to their partner's intake ${ }^{(23,24)}$. Salvy et al. ${ }^{(25)}$ reported that female and mixed-sex dyads exhibited significant modelling but there was no modelling in male dyads. Salvy et $a l^{(26,27)}$ have also conducted a number of dyad studies in normal-weight and overweight children. A good predictor of how much one dyad member eats is the amount eaten by the other dyad member ${ }^{(26,27)}$.

\section{Intake modelling during main meals}

Few studies have examined food intake modelling during main meals. Hermans et al. ${ }^{(18)}$ examined modelling effects on breakfast consumption and found that the presence of a low intake confederate did not cause participants to eat less than they did in the presence of a high intake confederate. This finding was interpreted as evidence that meal-modelling effects may be less powerful than snack intake modelling. It is possible that individuals have preexisting conceptions about how much they should eat for main meals but are more uncertain about snacking. The only other mealtime food intake modelling study we are aware of is by the same authors. Hermans et al. ${ }^{(28)}$ also tested modelling during an evening meal. Pairing participants with a high intake model resulted in greater meal intake by approximately $310 \mathrm{~kJ}(75 \mathrm{kcal})$ than pairing with a low intake model. Although statistically significant, this $15 \%$ difference in food intake is smaller than that usually observed in snacking studies. Yet, there has been no comparison of modelling effects in snacks $v$. main meals in the same study, so it is difficult to draw conclusions regarding differences between the two meal types.

\section{What drives intake modelling: high or low eaters?}

Recent studies do not provide strong support for the suggestion that a high intake confederate increases food intake relative to eating alone, but the effect of a low intake confederate appears to be more robust ${ }^{(18,19)}$. It may be that food intake modelling effects are driven primarily by self-presentation concerns and avoidance of being seen as a 'greedy' eater in front of a partner eating little or nothing. More direct of testing of the proposition that high eating partners liberate individuals from 
restraining their intake and thus may contribute to the spread of obesity though social networks is required ${ }^{(29)}$.

\section{Modelling of food choices}

Robinson \& Higgs ${ }^{(30)}$ tested whether a confederate choosing predominantly high-energy food items from a lunch buffet influenced the food selection of participants. Relative to a choosing-alone condition, and a condition in which a confederate chose predominantly low-energy food items (including vegetables), the presence of the high-energy confederate resulted in participants choosing and consuming up to $40 \%$ lower amount of vegetables. Two studies by McFerran et al. ${ }^{(31)}$ provide evidence that the amount of food individuals choose to serve themselves can be influenced by confederates choosing large and small amounts of food. McFerran et al. ${ }^{(31)}$ also manipulated the body size of confederates in their studies. Both slim and overweight confederates influenced food selections. Compared with a choosing-alone control condition, the presence of a slim confederate who selected a large amount of food increased the amount of snack food selected by the participants by 70 and $241 \%$. The presence of an overweight confederate who selected a large amount increased participant selections by 25 and 168\%. Finally, outside of the laboratory, Guarino et al. ${ }^{(32)}$ reported findings from an observational study in a canteen. Being accompanied by others who chose dessert increased the likelihood of the participant also choosing to eat dessert. It should be noted that not all studies examining modelling of food choices have found significant effects. Pliner \& Mann ${ }^{(33)}$ found little evidence that participants would choose a low-energy and unpalatable cookie over a higher-energy palatable cookie after learning that other participants had chosen the former. Thus, although in the majority of studies the modelling effect is observed, there are likely to be some barriers that prevent modelling (for example, when the available food in question is disliked).

\section{What factors influence modelling?}

The exact mechanisms underlying modelling effects are not entirely clear, although we suggest that they are driven mainly by social desirability. There have been two main suggestions as to why the eating behaviour of present diners influences food consumption: (1) appropriateness norms; (2) ingratiation concerns. Although these two explanations are likely to be closely linked, they produce distinct hypotheses that should help us understand when and how individuals will influence each other's eating behaviour.

\section{Modelling for appropriateness}

Herman et al. ${ }^{(8)}$ propose a normative model, which suggests that the amount eaten by a confederate constitutes an appropriate amount of food to eat that should not be exceeded.
Participants avoid eating more than this amount so as not to appear greedy. In support, individuals do not like to eat more than other diners because there are negative stereotypes regarding overeating ${ }^{(34)}$. The appropriateness account also suggests that modelling should be more likely in eating contexts that promote uncertainty about how much to eat. As noted, during main meals, for which individuals have clear scripts about what they normally eat, modelling effects are not particularly strong ${ }^{(18,21)}$. On the other hand, in settings where there is more uncertainty over appropriate eating behaviour, such as snacking on candy whilst watching television or during the taste-test paradigm, modelling effects are robust ${ }^{(15,21)}$. One implication of the appropriateness account is that modelling should be less strong when eating in an unfamiliar situation or with a dissimilar dining companion (for example, a short slim women eating with a heavy man). This is because the eating behaviour of a dissimilar eating companion would not be deemed 'appropriate' to model. Indeed, similarity between a participant and confederate in terms of body size promotes modelling ${ }^{(19)}$, although dissimilarity does not necessarily eliminate it ${ }^{(31)}$, suggesting that additional accounts are needed to explain modelling effects.

\section{Modelling for ingratiation}

Modelling effects may be explained in part because diners eat the same amounts as their partner to be liked ('imitation is the sincerest form of flattery'). Hermans et al. ${ }^{(17)}$ tested this proposition indirectly by instructing a confederate to act in a friendly $v$. unsociable manner. In the condition in which the confederate was friendly there was no evidence of modelling. Thus, when there was little desire to be liked (because the confederate was already accepting), the modelling effect was removed. Conversely, when the confederate was not sociable, a modelling effect was observed. We recently tested whether making individuals feel socially accepted before eating with a confederate would reduce the similarity in intake between the participant and the confederate. In line with the findings of Hermans et al. ${ }^{(17)}$ we found that there was less modelling in a condition that encouraged social acceptance than in a neutral control condition $^{(24)}$. Furthermore, the matching of intake in dyads is reported to be moderated by the desire to be liked by others and the tendency to consider the viewpoint of others ${ }^{(24)}$, as well as the extent to which individuals express themselves in social situations ${ }^{(35)}$.

The ingratiation account of modelling suggests that the extent to which an individual is keen to be liked will increase the likelihood that they copy their dining partner ${ }^{(24)}$. Similarly, eating situations in which individuals are keen to make a good impression (amongst new acquaintances, for example) should also increase the likelihood of modelling. What the ingratiation account predicts for modelling amongst friends or spouses is less clear. One hypothesis could be a reduced modelling effect, because partners 
would not feel the need to portray a positive image for those they are already close to. Conversely, if there were a wish to maintain a bond and be viewed positively by friends, modelling effects would be expected. Few studies have examined modelling in friends $v$. strangers and the evidence is mixed, with some studies finding a similar modelling effect in friends and strangers and others finding stronger modelling in friends than strangers ${ }^{(25,36)}$.

Work on unconscious mimicry may also support the modelling for ingratiation account. Individuals automatically mimic the gestures and hand movements of others $^{(37,38)}$. One suggestion why individuals mimic each other's eating behaviour is that it is an unconscious attempt to make the other individual like them because mimicry eases social interactions ${ }^{(37,38)}$. Hermans et al. ${ }^{(39)}$ examined this proposition by analysing video data from a confederate modelling study. They found that in $10 \mathrm{~s}$ time-gaps after a confederate had picked up a piece of food, the likelihood that a participant would also pick up food was increased. Sip taking during social alcohol drinking follows a similar pattern of results ${ }^{(40)}$ and self-report measures of social desirability predict alcohol modelling ${ }^{(41)}$.

\section{Modelling for reasons unrelated to appropriateness or ingratiation?}

Unconscious mimicry may occur in the absence of ingratiation attempts. It has been reported that viewing another individual performing an action (for example, grasping for food) activates a similar reaction in an individual's motor system ${ }^{(42,43)}$. Moreover, it has been suggested that such mimicry could occur regardless of salient goals or intentions ${ }^{(44)}$. If mimicry underlies modelling, then modelling of food picking would be predicted to occur immediately after the action of a high or low intake model. Hermans et al. ${ }^{(39)}$ reported that modelling was more likely within $10 \mathrm{~s}$ of a model picking up food, but, as far as we are aware, there have been no investigations of modelling within shorter time frames.

Beliefs about the types and amount of food that other individuals eat have profound effects on eating behaviour, even when those other individuals are not present, suggesting that the effects cannot be driven solely by ingratiation $^{(45,46)}$. Roth et al. ${ }^{(46)}$ and Pliner \& Mann ${ }^{(33)}$ examined the effect of providing bogus information about what non-present others ate in an experiment. This design is commonly referred to as the 'remote-confederate' design. Before giving participants the opportunity to snack on a high-fat and -sugar snack food (cookies), the researchers exposed participants to fictional information about what other individuals had eaten during earlier sessions. When participants believed that other individuals in the study had eaten large amounts of the snack, their own intake increased substantially ${ }^{(33,46)}$. In a similar study by Feeney et $a l .{ }^{(20)}$, when participants were led to believe that the norm was to eat very little, they more than halved their intake of pizza and ate $840 \mathrm{~kJ}$ (200 kcal) less than participants in a no-norm control. Using a remote-confederate design Robinson et al. $^{(47)}$ found bidirectional effects of norm information: participants in a high-norm condition ate significantly more (approximately $40 \%$ or $310 \mathrm{~kJ}$ (75 kcal)) than in a no-norm condition, whereas participants in a low-norm condition ate significantly less than the nonorm group (approximately 40\%).

There is also research indicating that food intake modelling can occur in adults and children as a result of watching a non-present individual's eating behaviour ${ }^{(45)}$. Romero et al. ${ }^{(48)}$ examined whether viewing a video of another child selecting and eating a small or large number of cookies influenced selection and consumption of cookies in children aged 8-12 years who were participating in the same study. Healthy-weight and overweight girls were recruited. More cookies were eaten when watching another child select and eat a large amount of cookies than when watching another child eat a small amount (a $389 \mathrm{~kJ}$ (93 kcal) difference between conditions). The interaction between participant weight status and amount chosen by the video model was only marginally significant, but the effect was particularly pronounced in overweight children: a $682 \mathrm{~kJ}$ (163 kcal) difference between conditions for the overweight children $v$. a $184 \mathrm{~kJ}$ ( $44 \mathrm{kcal}$ ) difference for the healthy-weight children. The finding that a model can influence intake when participants believe that they will never meet that individual and when they believe that their eating behaviour is not being monitored is of interest because it suggests that social desirability concerns are unlikely to motivate mimicry. Although this could be perceived as evidence that automatic mimicry is occurring, the design of such studies could accommodate a further possibility: that a model's eating behaviour provides information about how most other individuals behave and creates a perceived eating norm.

\section{Perceived eating norms}

Perceived eating norms refer to individuals' beliefs about how others around them behave (also known in other areas of psychology as 'descriptive norms'). The notion that beliefs about the behaviour of those around us influence how we choose to behave is integral to sociocognitive models of health behaviour. In particular, although not included in the originally proposed model, descriptive norms are now recognised as a key predictor variable of intentions and behaviour in the theory of planned behaviour ${ }^{(49)}$ (an extension of what was the theory of reasoned action). Using this model of behaviour, a number of researchers have shown that descriptive norms directly inform behavioural intentions, which then shape actual behaviour ${ }^{(49-51)}$. Making use of meta-analysis to synthesise a large number of correlational studies, Rivis \& Sheeran ${ }^{(52)}$ showed that descriptive norms are predictive of behavioural intentions and they exert a stronger 
influence than injunctive norms (also referred to as subjective norms), which are beliefs about the social acceptability of behaviour. These findings underline the importance of differentiating between the influences of different types of norms when explaining health-relevant behaviours. In relation to eating behaviours, studies guided by the theory of planned behaviour have confirmed the association between perceived eating norms and eating behaviour $^{(53,54)}$. The theory of planned behaviour suggests that individuals look outwards to the behaviour of others and modify their behavioural intentions to conform to the perceived norm ${ }^{(52)}$.

Additional evidence for the relationship between perceived eating norms and behaviour comes from the work of Lally et al. ${ }^{(55)}$ who examined habitual intake of adolescents using a large survey study design. They measured habitual intake of fruits and vegetables, sugar-sweetened drinks and 'unhealthy snacks' alongside beliefs about peers' habitual intake (a perceived eating norm). Perceived eating norms were strongly associated with habitual intake. The measures of perceived eating norms explained between 17 and $22 \%$ of the variance in habitual intake of the foods examined. Using a similar cross-sectional design, Perkins et al. ${ }^{(56)}$ examined the habitual intake of sugar-sweetened beverages of 4000 high school students in the USA. Self-reported habitual intake was predicted by perceived peer norms; if participants believed their peers drank a large number of sugarsweetened beverages this increased the likelihood that they would also be consuming a large amount. These findings were interpreted in line with a pluralistic ignorance account in which individuals assume that most other people eat unhealthily, when they in fact do not ${ }^{(57)}$. Both studies by Lally et $a l .{ }^{(55)}$ and Perkins et $a l .{ }^{(56)}$ support this idea, as participants tended to believe that their peers ate fewer vegetables and drank more soda than they actually did.

Another study ${ }^{(58)}$ examined the predictive power of perceived social norms in a group of over 3000 female adults in Australia using a cross-sectional design and assessment of consumption of fruit and vegetables, soft drinks and fast food. Perceived social norms predicted habitual behaviour for all food types. As well as habitual intake of food, more general intentions to eat a healthy diet are influenced by perceived peer norms. A further study ${ }^{(59)}$ has shown that the perceived eating norms of peers predicted healthy eating intentions of young adults (23\% of variance explained). However, this effect was observed only in participants who identified with their peer group. An issue with this type of cross-sectional data is a possible false consensus effect, whereby individuals might presume that their behaviour is representative of what other people do ${ }^{(60)}$. Therefore, correlational data showing that norms and behaviour are associated could be potentially explained by an individual's behaviour influencing their perceptions of the norm. However, there is also now a strong body of evidence that has involved manipulating eating norms and examining the effect on eating behaviour, to more directly examine causality.

\section{Manipulating context-specific perceived eating norms}

Burger et al. ${ }^{(61)}$ report two studies in which participants made a food choice and were provided with information suggesting that other individuals had chosen a low-energy 'light' snack bar or high-energy 'full-fat' snack bar. In study 1 , the energy difference between the two snack bar types was up to $710 \mathrm{~kJ}$ ( $170 \mathrm{kcal})$. Both studies found that the participants who were led to believe that the norm was to select the high-energy snack bar were far more likely to select a high-energy snack bar than participants led to believe that the norm was to select a low-energy snack bar. In study 1 , the high-energy-choice norm resulted in $28 \%$ more of participants selected the higher-energy option and in study 2 the increase was $35 \%^{(61)}$.

Mollen et al. ${ }^{(62)}$ have also shown that norms guide food choices in a field experiment. Participants were exposed to social-norm messages in a food court. Participants were more likely to choose 'healthier' food options after exposure to a norm message emphasising healthy eating, than after exposure to a no-norm control message. Similarly, Salmon et $a l .^{(63)}$ have reported that individuals are more likely to choose a healthy food option after it was suggested to them that this choice was endorsed by the majority of other individuals. This effect was more pronounced for individuals lacking self-control. The authors suggested that the norms provided a form of decision-making heuristic (or 'behavioural guide'). Moving outside of the laboratory, Prinsen et al. ${ }^{(64)}$ have also shown that healthy food choices are more likely when aspects of the environment imply that it is the norm to choose this option.

The duration of perceived norm effects on intake has been less well investigated. Feeney et al. (JR Feeney, P Pliner, J Polivy and PC Herman, unpublished results) used a repeated-measures design in which participants were exposed to a high- or low-intake norm before eating and then returned for three further sessions on consecutive days. There was no eating-norm information present in these later sessions. A significant eating-norm effect on pizza intake was observed during the first session. The difference in intake between the low- and high-intakenorm conditions was approximately $1260 \mathrm{~kJ}$ (300 kcal), confirming the strong effect of perceived eating norms on food intake. Significant differences were also observed for the following two sessions when the eating-norm information was not present. Although the results were in the expected direction, by the third session the difference in intake between norm conditions was sizeable $(710 \mathrm{~kJ}(170 \mathrm{kcal}))$, but no longer statistically significant. One reason why the influence of a perceived eating norm decreased by the final session might be that repeated daily consumption of the test food resulted in some participants habituating to the food, weakening the influence 
of a high-intake norm ${ }^{(65)}$. Nevertheless, these findings indicate that perceived eating norms can have both immediate and persistent influences on food intake.

\section{Experimentally manipulating non-context-specific eating norms}

Other studies have examined whether perceived eating norms that are not context specific produce similar effects on the types and amounts of food that individuals eat. We recently conducted two studies in which university students viewed flyers and posters about the daily fruit and vegetable intake of other students ${ }^{(66)}$. Participants either saw materials emphasising the health benefits of eating fruit and vegetables or information about fruit and vegetable eating norms. We used real survey data to suggest that other individuals (students from the same university) eat more portions of fruit and vegetables on a daily basis than might be expected. In an apparently unrelated later part of their visit to the laboratory, participants selected and consumed a lunch (study 1) or a snack (study 2). In study 1, after being exposed to an eating-norm message about vegetable intake, usually low consumers of vegetables ate more vegetables than after exposure to health message. Compared with the participants shown information about the health benefits of eating vegetables, they more than doubled their intake to $67 \mathrm{~g}$, which is approximately one recommended daily serving. There was also a proportional increase in the percentage of the meal derived from vegetables. There was no effect of the manipulation on habitual high consumers of vegetables and this is probably because they were already adhering to the presented eating norm and did not feel motivated to change their behaviour ${ }^{(66)}$. In study 2 , the eating-norm information that was provided emphasised that other students eat more portions of fruits and vegetables than might be expected. Food choice and intake during a snack were later measured. A similar pattern of results was observed as for study 1 . Usually high consumers of fruits and vegetables consumed a relatively large amount of fruits and vegetables regardless of seeing eating-norm or health information. In usually low consumers of fruits and vegetables, a three-fold increase was observed after the social $v$. the health information and this approximated to consuming an extra recommended daily serving. The increase in fruit and vegetable intake was also associated with a decrease in consumption of high-energy snack foods (to accommodate the additional fruit and vegetables), resulting in the eating-norm message condition consuming 318 fewer $\mathrm{kJ}$ (76 fewer kcal) than the health information control condition. There was no effect of a message stating that other individuals approve of eating fruit and vegetables and we turn to the significance of this finding shortly.

Three other studies have examined whether information about eating norms influences fruit and vegetable intake.
Croker et al. ${ }^{(67)}$ exposed participants to survey information suggesting that UK residents have high intentions to consume their five portions of fruits and vegetables per $\mathrm{d}$. In participants with low intentions at baseline (males), exposure to this information was associated with greater intended fruit and vegetable consumption than exposure to control messages. Stok et al. ${ }^{(68)}$ also examined the effect of exposing participants to eating norms about fruit intake on intentions to eating fruit. Participants were either led to believe that other students eat a lot of fruit or very little. Intentions to consume fruit during the following week were then measured. Participants exposed to a high fruit intake norm intended to consume significantly more portions of fruit during the following week than participants exposed to the low-fruit-intake-norm message. Following up these results in a second study, the authors tested whether norm messages increased self-reported fruit intake. Participants were aware that the study was designed to increase fruit consumption. Participants were given some dietary advice and then kept a food diary for 1 week. During this time they were exposed intermittently to messages emphasising that others eat a lot or very little fruit. The norm reference group was either other students or the national population. Although there was no effect of a national population norm, students exposed to high fruit intake norms about other students self-reported that they ate on average four more portions of fruit than students who were exposed to low-fruitintake-norms messages. This is a moderate effect but it should be noted that participants were already attempting to increase their fruit intake, so an important implication is that norms may influence the individuals who are already motivated to change their diet.

\section{Using perceived eating norms to reduce intake of 'unhealthy' foods}

Perceived eating-norm messages have also been shown to reduce the selection and intake of unhealthy foods. In a recent study, we exposed participants either to a message that most university students try to limit the amount of 'junk food' they eat or to a control message. We then examined the effect of this manipulation on snack choice and intake $^{(69)}$. Junk food was defined as food that is high in energy with little nutritional value. Exposure to the eating-norm message resulted in a $28 \%$ reduction in the weight ( $\mathrm{g}$ ) of high-energy snack foods eaten (chocolate, cookies and crisps) relative to the control message (a reduction of about $250 \mathrm{~kJ}(60 \mathrm{kcal}))^{(69)}$. Berger \& Rand $^{(70)}$ and Berger \& Heath ${ }^{(71)}$ examined whether eating norms about an undesirable social group that individuals would not want to associate with influenced food choices and intake. Exposing participants to an eating-norm message suggesting that an undesirable group regularly ate 'junk food' resulted in participants reducing their 'junk food' intake, in particular energy from fat ${ }^{(70,71)}$. 


\section{Effect of norm types}

It should be noted that some studies have assessed the predictive power of perceived eating norms on dietary behaviour but found no effect ${ }^{(72)}$. This may be because the conceptualisation of what constitutes a perceived eating norm varies across studies. A descriptive norm refers to beliefs about how others behave, whereas an injunctive norm refers to beliefs about approval of behaviours. Injunctive norms are not always predictive of behaviour. Conner et al. ${ }^{(73)}$ found no evidence of the predictive power of injunctive norms, whereas another study ${ }^{(74)}$ found that injunctive norms predicted intentions towards consuming GM food. Similar inconsistencies are observed in studies examining whether injunctive norms predict habitual fruit and vegetable intake ${ }^{(75)}$. This pattern of results may suggest that perceptions about what other individuals are actually eating are a stronger predictor of eating behaviour than perceptions of what other individuals think should be eaten. This perhaps comes as no surprise, but additional aspects of three of the studies we reviewed earlier support this proposition. Lally et al. ${ }^{(55)}$ found that perceptions of peers' attitudes towards consuming different food types did not predict intake, whereas perceptions of what peers were eating were predictive. Mollen et al. ${ }^{(62)}$ too found no evidence that an injunctive norm increased healthier food intake and we have also found that a message about approval of fruit and vegetable consumption did not affect eating behaviour ${ }^{(66)}$.

\section{When will perceived eating norms influence dietary behaviour?}

The literature to date has focused on the role of perceived eating norms on intake of 'junk foods' and intake of fruits and vegetables. The high fat, sugar and salt content of the former and high fibre and phytonutrient content of the latter mean that such findings have obvious nutritional relevance. However, further work is needed to understand the mechanism(s) that underlie the effect of perceived eating on dietary behaviour. We suggest that social desirability is unlikely to be a major factor affecting usual eating patterns. Instead, we suggest two separate accounts: one centred on norms acting as useful information about how best to behave and the other centred on the expression of social identity. It seems unlikely that individuals follow eating norms simply because they are concerned about feeling socially accepted. In most of the laboratory studies discussed here, such an explanation is not sufficient, because perceived eating norms have large effects on dietary behaviour even when participants eat alone or are unaware that their behaviour is being monitored.

\section{Perceived eating norms as behavioural guides}

As has been theorised for other types of behaviour (see Rimal et al. ${ }^{(76)}$ and Lapinski \& Rimel $^{(77)}$ ), perceived eating norms may act as a behavioural guide for perceived utility - 'everyone else is behaving this way for a reason so it is probably a good idea for me to behave similarly'. An example of this is that information about low cancer screening rates causes individuals to be less motivated to be screened, presumably because learning that others are not concerned about screening reduces the personal importance of screening ${ }^{(78)}$. This account may be well placed to explain why perceived norms influence intake of foods that are typically thought of as 'healthy' (fruits and vegetables) and 'unhealthy' (fast food, snack food) and may also explain why perceived eating norms influence eating behaviours even when social acceptance concerns are low. This account is also in line with earlier theoretical accounts for the role of perceived norms in decision making, as outlined in recent adaptations to the theory of planned behaviour ${ }^{(49)}$ and work in social psychology ${ }^{(79)}$.

If social norms act as a behavioural guide, then the behaviour of socially proximal individuals should be more likely to be followed than the behaviour of socially distant individuals. In support, proximity predicts the extent to which perceived social norms influence drinking behaviour $^{(80)}$. In a study by Cruyws et $a l .{ }^{(81)}$ participants learnt about the amount of food an individual from a socially proximal group had eaten (other students at their university), or about an individual from a less proximal group (students from a rival university). The perceived eating norm influenced intake in the former but not the latter condition. Furthermore, when perceived eating norms have been reported to predict habitual dietary behaviour, the norms have been about one's immediate peers ${ }^{(59,68)}$. However, it should be noted that norms about groups that are not socially proximal can still influence behaviour. Perceived norms about an entire national population influence health behaviour intentions ${ }^{(67,78)}$. Croker et $a l{ }^{(67)}$ found that a message emphasising that individuals in the UK intend to eat large amounts of fruits and vegetables increased intentions to consume fruits and vegetables.

\section{Perceived eating norms and identity signalling}

A recent suggestion that may account for influence on health behaviour of socially distant group norms is centred on social group membership and 'identity signalling, ${ }^{,(70,82)}$. The extent to which an individual identifies with a group may affect whether they behave in line with their perceptions of how that group eats and this may be independent of degree of social proximity to the group. Considering the study by Croker et $a l^{(67)}$, the participants presumably identified themselves as UK citizens and this may have influenced their intentions to eat fruit and vegetables. In line with this reasoning, Berger \& Rand ${ }^{(70)}$ and Berger \& Heath $^{(71)}$ found that in some circumstances individuals will be motivated to dissociate themselves from a social group by changing their food choices. Similarly, social 
group identity predicts intentions to follow nutritional guidelines for salt intake ${ }^{(82)}$. Social group membership and identity signalling may also account for reports that immigrants adopt the stereotypical eating habits of their new nation. Guendelman et al. ${ }^{(83)}$ reported that a desire to prove they belong in America motivated US immigrants to consume more typically American food (for example, fast food). Thus, building on the work of Berger \& Rand ${ }^{(70)}$, eating norms may be particularly influential on behaviour if there is a strong motivation to associate oneself with the referent group. According to this explanation we would predict that individuals who have a strong sense of national pride would be influenced by their perceptions of national eating norms or cuisine.

\section{General discussion}

It is clear that both adults and children are influenced by their perceptions of other individuals' eating behaviours. The perceived behaviour of others can increase or decrease consumption of both high-energy 'junk foods' and health-protective foods. In line with the distinction proposed by Deutsch \& Gerard ${ }^{(79)}$, we suggest that a convincing body of evidence has now amassed indicating that social influence on eating occurs for two distinct reasons: the behaviour of other individuals serves as adaptive information to follow (a form of 'behavioural guide' or 'informational influence'), and eating behaviour can be a way to ingratiate oneself with others or signal identity ('normative influence'). Based on these observations, specific hypotheses can be made to intervene and improve nutrition.

\section{Applied relevance: perceived eating-norm messages}

In line with socio-cognitive models of health behaviour and norm theories in social psychology ${ }^{(52,77,84)}$, perceived eating norms appear to influence behaviour by acting as a form of behavioural guide. Thus, public health messages could emphasise the healthy eating habits of other individuals or minimise the perception that other individuals are eating unhealthily. How best to formulate these messages should be driven by our understanding of the underlying mechanism(s). For example, messages outlining the behaviour of others (rather than the attitudes or intentions of others) would be expected to be most effective because this information may serve as the best adaptive cue. In addition, messages about individuals for whom a social connection is lacking may be easily dismissed as an inappropriate guide. Furthermore, if a norm suggests implicitly that a significant proportion of individuals choose not to follow that norm (for example, 'did you know that 38\% of people consume five portions of fruit and vegetables a day' also can be interpreted as $62 \%$ of people choose not to), this could have the opposite of the desired effect and encourage those already adhering to the presented norm to eat less healthily.

The majority of studies to date have examined contextspecific norms ('what other people do in this experiment') and the effect sizes observed are substantial. Thus, contextrelevant point of choice or purchase social-norm messages may be hypothesised to be particularly effective in changing behaviour ('most people in this canteen have vegetables with their lunch'). Context-specific norms can presumably produce a clear behavioural guide that reduces ambivalence over whether or not individuals view themselves as adhering to or rejecting a norm. In contrast, a non-context-specific-norm message ('most people eat three portions of vegetables on a daily basis') may not commit consumers to act immediately on exposure to the norm message. If the norm is not tied to a specific context or setting, individuals could choose not to eat vegetables during their lunch without rejecting the norm.

\section{Applied relevance: positive social image and healthy eating}

Tarrant $\&$ Butler ${ }^{(82)}$ and Berger $\&$ Rand ${ }^{(70)}$ suggest that social influence on eating behaviour can occur because of aspirations regarding affiliation or identity. In short, individuals will conform to the eating behaviour of others because they wish to portray a positive image ${ }^{(61,62)}$. Making use of this knowledge to encourage adolescents to make healthier dietary choices could be fruitful. Adolescence is a period of life in which desires to be liked and 'fit in' to peer groups are high $^{(85,86)}$. One possibility is to extend traditional healthy eating interventions and use campaigns led by aspirational figures, such as children in older school years, or more specifically, adolescents who are perceived as the 'cool' in the crowd. In a similar vein, school-based interventions that use media to encourage adolescents to associate healthy eating behaviours with desirable social groups warrant further investigation ${ }^{(87,88)}$.

Moving away from working directly with adolescents, another possibility is to embed healthy eating habits within social identity. For example, university students tend to base their sense of self on their position as a student of their specific institute. Similarly, sports fans can have a strong sense of affiliation to their respective teams. Thus, approaches that aim to pair identities with healthy eating could motivate individuals to eat more healthily in order to maintain identity. Pairing healthier eating with desirable groups rather than pairing unhealthy eating with undesirable groups may be more feasible and less offensive to the social group deemed undesirable.

\section{Future research}

In the present review we have drawn attention to the important influence of social context on eating behaviour, as well as highlighting underlying mechanisms that may be 
relevant for nutritional interventions. However, a number of questions remain unanswered. Changes to societal norms have been proposed as one mechanism by which overeating and obesity have become commonplace ${ }^{(89)}$. Although the literature reviewed here suggests that eating norms can shape food choice and intake, how norms are transferred through large social groups remains unclear. Social proximity may predict the norms that individuals will follow and would include the normative behaviour of siblings, parents, spouses, friends and work colleagues. Hence, it will be critical to understand the factors that determine whose norms we follow and why.

We will also benefit from a better understanding of how broader eating norms make an impact on behaviour. Information about other individuals in the UK can motivate intentions to eat more healthily ${ }^{(67)}$. Similarly, it has been reported that making a national identity more salient results in changes to dietary intentions ${ }^{(82)}$. If eating-norm messages or interventions prove to be feasible, broad messages would have the potential to reach larger groups of individuals in need of intervention. It will be important to examine the effectiveness of different types of message and the underlying mechanisms.

Better understanding of the drivers of eating in social situations is also required. There are several potential underlying mechanisms: ingratiation concerns; appropriateness; updating of perceived eating norms; unconscious mimicry. Picking apart these mechanisms is not easy but in future it would be informative to test their relative mediating roles. For example, we have reported recently that social information influences how much an individual believes they like a food ${ }^{(90)}$. Thus, it could be that some social influence effects on behaviour occur in part because social information changes beliefs about the self and individuals then change their behaviour to maintain a sense of consistency between their self-concept and behaviour.

A major limitation of much of the social influence research to date has been an emphasis on sampling young adult healthy-weight females. Only a small number of studies have examined modelling in males ${ }^{(25,91)}$. Moreover, as the population weight norms are changing, if social influence inspired interventions for dietary behaviours are to be effective, we will also need to understand whether overweight and obese individuals respond in a similar way to eating norms as healthy-weight individuals. One prediction is that obese individuals may categorise themselves and their eating as 'different' from a general population, so they may disregard norms about the eating behaviour of most other individuals. Alternatively, there has long been a suggestion that obese individuals are more prone to external eating cues ${ }^{(92)}$ and one might predict enhanced responsiveness to information about perceived norms. Although weight gain is likely to be under social influence $^{(29)}$, further evidence is required that social norms about eating contribute to unhealthy dietary practices and the development of obesity.

\section{Conclusions}

Social context has a substantial impact on the amounts and types of food that individuals eat. There are multiple mechanisms explaining social influences on eating. Building on existing models of behaviour and recent experimental evidence, we suggest that harnessing these influences has great potential in promoting healthier nutrition.

\section{Acknowledgements}

There are no relevant funding or sponsorship disclosures from any of the authors. E. R., J. B. and S. H. wrote the paper. The authors declare no conflicts of interest. There are no relevant competing interests from any of the authors.

\section{References}

1. Swinburn BA, Sacks G, Hall KD, et al. (2012) The global obesity pandemic: shaped by global drivers and local environments. Lancet 378, 804-814.

2. Lobstein $\mathrm{T} \&$ \& Dibb S (2005) Evidence of a possible link between obesogenic food advertising and child overweight. Obes Rev 6, 203-208.

3. Levitsky DA, Halbmaier CA \& Mrdjenovic G (2004) The freshman weight gain: a model for the study of the epidemic of obesity. Int J Obes 28, 1435-1442.

4. De Castro JM, Brewer ME \& Elmore DK (1990) Social facilitation of the spontaneous meal size of humans occurs regardless of time, place, alcohol or snacks. Appetite 15, 89-101.

5. De Castro JM \& Brewer ME (1992) The amount eaten in meals by humans is a power function of the number of people present. Physiol Behav 5, 121-125.

6. Pliner P, Bell R, Hirsch ES, et al. (2006) Meal duration mediates the effect of social facilitation on eating in humans. Appetite 46, 189-198.

7. Cavazza N, Graziani AR \& Guidetti M (2011) Looking for the 'right' amount to eat at the restaurant: social influence effects when ordering. Soc Influ 6, 274-290.

8. Herman CP, Roth DA \& Polivy J (2003) Effects of the presence of others on food intake: a normative interpretation. Psychol Bull 129, 873-886.

9. Conger JC, Conger AJ, Costanzo PR, et al. (1980) The effect of social cues on the eating behavior of obese and normal subjects. J Pers 48, 258-271.

10. De Luca R \& Spigelman M (1979) Effects of models on food intake of obese and non-obese female college students. Can J Behav Sci 11, 124-129.

11. Nisbett RE \& Storms MD (1984) Cognitive and social determinants of food intake. In Thought and Feeling: Cognitive Alteration of Feeling States, pp. 196-208 [H London and RE Nisbett, editors]. Chicago: Aldine.

12. Goldman SJ, Herman CP \& Polivy J (1991) Is the effect of a social model on eating attenuated by hunger? Appetite 17, 129-140.

13. Rosenthal B \& Marx RD (1979) Modeling influences on the eating behavior of successful and unsuccessful dieters and untreated normal weight individuals. Addict Behav $\mathbf{4}$, $215-221$.

14. Rosenthal B \& McSweeney FK (1979) Modeling influences on eating behavior. Addict Behav 4, 205-214. 
15. Johnston L (2002) Behavioral mimicry and stigmatization. Soc Cognition 20, 18-35.

16. Brunner TA (2010) How weight-related cues affect food intake in a modeling situation. Appetite 55, 507-511.

17. Hermans RCJ, Engels RCME, Larsen JK, et al. (2009) Modeling of palatable food intake. The influence of quality of social interaction. Appetite 52, 801-804.

18. Hermans RCJ, Herman CP, Larsen JK, et al. (2010) Social modeling effects on young women's breakfast intake. $\mathrm{J} \mathrm{Am}$ Diet Assoc 110, 1901-1905.

19. Hermans RC, Larsen JK, Herman CP, et al. (2010) Modeling of palatable food intake in female young adults. Effects of perceived body size. Appetite 51, 512-518.

20. Feeney JR, Polivy J, Pliner P, et al. (2011) Comparing live and remote models in eating conformity research. Eat Behav 12, $75-77$.

21. Hermans RC, Larsen JK, Herman CP, et al. (2009) Effects of social modeling on young women's nutrient-dense food intake. Appetite 53, 135-138.

22. Bevelander KE, Anschutz DJ \& Engels RCME (2012) Social norms in food intake among normal weight and overweight children. Appetite 58, 864-872.

23. Herman CP, Koenig-Nobert S, Peterson J, et al. (2005) Matching effects on eating: do individual differences make a difference? Appetite 45, 108-109.

24. Robinson E, Tobias T, Shaw L, et al. (2011) Social matching of food intake and the need for social acceptance. Appetite 56, 747-752.

25. Salvy SJ, Jarrin D, Paluch R, et al. (2007) Effects of social influence on eating in couples, friends and strangers. Appetite 49, 92-99.

26. Salvy SJ, Romero N, Paluch R, et al. (2007) Peer influence on pre-adolescent girls' snack intake: effect of weight status. Appetite 49, 177-182.

27. Salvy SJ, Howard M, Read M, et al. (2009) The presence of friends increases food intake in youth. Am J Clin Nutr 90 , $282-287$.

28. Hermans RC, Larsen JK, Herman CP, et al. (2012) How much should I eat? Situational norms affect young women's food intake. Br J Nutr 107, 588-594.

29. Christakis NA \& Fowler JH (2007) The spread of obesity in a large social network over 32 years. $N$ Engl J Med 357, $370-379$.

30. Robinson E \& Higgs S (2013) Food choices in the presence of 'healthy' and 'unhealthy' eating partners. Br J Nutr 109, $765-771$.

31. McFerran B, Dahl D, Fitzsimons G, et al. (2010) I'll have what she's having. Effects of social influence and body type on the food choices of others. J Consum Res 36, 915-929.

32. Guarino M, Fridrich P \& Sitton S (1995) Male and female conformity in eating behavior. Psychol Rep 75, 603-609.

33. Pliner P \& Mann N (2004) Influence of social norms and palatability on amount consumed and food choice. Appetite 42, 227-237.

34. Vartanian LR, Herman CP \& Polivy J (2007) Consumption stereotypes and impression management: how you are what you eat. Appetite 48, 265-277.

35. Brunner TA (2012) Matching effects on eating. Individual differences do make a difference! Appetite 58, 429-431.

36. Salvy SJ, Vartanian LR, Coelho JS, et al. (2008) The role of familiarity on modeling of eating and food consumption in children. Appetite 50, 514-518.

37. Chartrand TL \& Bargh JA (1999) The chameleon effect. The perception-behavior link and social interaction. J Pers Soc Psychol 76, 893-910.

38. Chartrand TL \& van Baaren R (2009) Human mimicry. $A d v$ Exp Soc Psychol 41, 219-274.
39. Hermans R, Lichtwarck-Aschoff A, Bevelander KE, et al. (2012) Mimicry of food intake: the dynamic interplay between eating companions. PLOS ONE 7, e31027.

40. Larsen H, Engels RCME, Souren PM, et al. (2010) Peer influence in micro-perspective: imitation of alcoholic and nonalcoholic beverages. Addict Behav 35, 49-52.

41. Caudill BD \& Kong FH (2001) Social approval and facilitation in predicting modeling effects in alcohol consumption. $J$ Subst Abuse 13, 425-441.

42. Rizzolatti G \& Craighero L (2004) The mirror-neuron system. Annu Rev Neurosci 27, 169-192.

43. Iacoboni M, Woods RP, Brass M, et al. (1999) Cortical mechanisms of human imitation. Science 286, 2526-2528.

44. Cook R, Bird G, Lünser G, et al. (2012) Automatic imitation in a strategic context: players of rock-scissors imitate opponents' gestures. Proc Biol Soc 279, 178-786.

45. Tanner RJ, Ferraro R, Chartrand TL, et al. (2007) Of chameleons and consumption: the impact of mimicry on choice and preferences. J Consum Res 34, 754-766.

46. Roth DA, Herman CP, Polivy J, et al. (2001) Selfpresentational conflict in social eating situations: a normative perspective. Appetite 36, 165-171.

47. Robinson E, Benwell H \& Higgs S (2013) Social norms can increase and decrease snack food intake in the absence of normative concerns. Appetite 65, 20-24.

48. Romero ND, Epstein LH \& Salvy SJ (2009) Peer modeling influences girls' snack intake. J Am Diet Assoc 109, 133-136.

49. Sheeran P \& Orbell S (1999) Augmenting the theory of planned behavior: roles for anticipated regret and descriptive norms. J Appl Soc Psychol 23, 2107-2142.

50. Grube JW, Morgan M \& McGree ST (1998) Attitudes and normative beliefs as predictors of smoking intentions and behaviors: a test of three models. BrJ Soc Psychol 25, 81-93.

51. White KM, Terry DJ \& Hogg MA (1994) Safer sex behavior. The role of attitudes, norms and control factors. J Appl Soc Psychol 24, 2164-2192.

52. Rivis A \& Sheeran P (2003) Descriptive norms as an additional predictor in the theory of planned behaviour: a meta-analysis. Curr Psychol 33, 218-233.

53. Povey R, Conner M, Sparks P, et al. (2000) The theory of planned behavior and healthy eating. Examining additive and moderating effects of social influence variables. Psychol Health 14, 991-1006.

54. Berg C, Jonsson I \& Conner M (2000) Understanding choice of milk and bread for breakfast among Swedish children aged 11-15 years. An application of the theory of planned behavior. Appetite 34, 5-19.

55. Lally P, Bartle N \& Wardle J (2011) Social norms and diet in adolescents. Appetite 57, 623-627.

56. Perkins JM, Perkins HW \& Craig DW (2010) Misperceptions of peer forms as a risk factor for sugar-sweetened beverage consumption among secondary school students. J Am Diet Assoc 110, 1916-1921.

57. Hines D, Saris RN \& Throckmorton-Belzer L (2006) Pluralistic ignorance and health risk behaviors: do college students misperceive social approval for risky behaviors on campus and in media? J Appl Soc Psychol 32, 2621-2640.

58. Ball K, Jeffrey W, Abbott G, et al. (2010) Is healthy behaviour contagious: associations of social norms with physical activity and healthy eating. Int J Behav Nutr Phys Act 7, 86.

59. Louis W, Davies S, Smith J, et al. (2012) Pizza and pop and the student identity: the role of referent group norms in healthy and unhealthy eating. J Soc Psychol 147, $57-74$

60. Grindle M \& Miller N (1987) Ten years of research on the false-consensus effect. An empirical and theoretical review. Psychol Bull 102, 72-90. 
61. Burger JM, Bell H, Harvey K, et al. (2010) Nutritious or delicious? The effect of descriptive norm information on food choice. J Soc Clin Psychol 29, 228-242.

62. Mollen S, Rimal R, Ruiter KA, et al. (2013) Healthy and unhealthy social norms and food selection. Findings from a field-experiment. Appetite 65, 83-89.

63. Salmon SJ, Fennis BM, de Ridder DT, et al. (2013) Health on impulse: when low self-control promotes healthy food choices. Health Psychol (epublication ahead of print version 11 March 2013).

64. Prinsen S, de Ridder DTD \& de Vet E (2013) Eating by example. Effects of environmental cues on dietary decisions. Appetite 70, 1-5.

65. Epstein LH, Temple JL, Roemmich JN, et al. (2009) Habituation as a determinant of human food intake. Psychol Rev 116, 384-407.

66. Robinson E, Fleming A \& Higgs S (2013) Prompting healthier eating: comparing the use of social norm and health based messages. Health Psychol (In the Press).

67. Croker H, Whitaker KL, Cooke L, et al. (2009) Do social norms affect intended food choice? Prev Med 49, 190-193.

68. Stok FM, de Ridder DT, de Vet E, et al. (2012) Minority talks: the influence of descriptive social norms on fruit intake. Psychol Health 27, 956-970.

69. Robinson E, Harris E, Thomas J, et al. (2013) Reducing junk food in young adults. A role for social norm and health based messages. Int J Behav Nutr Phys Act 10, 73.

70. Berger J \& Rand L (2008) Shifting signals to help health: using identity signalling to reduce risky health behaviors. J Cons Res 35, 509-518.

71. Berger J \& Heath C (2008) Who drives divergence? Identitysignalling, outgroup dissimilarity, and the abandonment of cultural tastes. J Pers Soc Psychol 95, 593-607.

72. Povey R, Conner M, Sparks P, et al. (2000) Application of the theory of planned behaviour to two dietary behaviours. Roles of perceived control and self-efficacy. $\mathrm{Br} J$ Health Psychol 5, 121-139.

73. Conner M, Norman P \& Bell R (2000) The theory of planned behavior and healthy eating. Health Psychol 21, 194-201.

74. Cook AJ, Kerr GN \& Moore K (2000) Attitudes and intentions towards purchasing GM food. J Econ Psychol 23 , $557-572$

75. Guillaumie L, Godin G \& Vézina-Im L (2010) Psychosocial determinants of fruit and vegetable intake in adult populations: a systematic review. Int J Behav Nutr Phys Act $\mathbf{7}$, $7-12$.
76. Rimal RN, Lapinski MK, Cook RJ, et al. (2005) Moving toward a theory of normative influences: how perceived benefits and similarity moderate the impact of descriptive norms on behaviors. I Health Commun 10, 433-450.

77. Lapinski MK \& Rimal RN (2005) An explication of social norms. Commun Theory 15, 127-147.

78. Sieverding M, Decker S \& Zimmermann F (2010) Information about low participation in cancer screening demotivates other people. Psychol Sci 21, 941-943.

79. Deutsch M \& Gerard H (1955) A study of normative and informational social influences upon individual judgment. J Abnorm Soc Psychol 51, 629-636.

80. LaBrie JW, Hummer JF, Neighbors C, et al. (2010) Whose opinion matters? The relationship between injunctive norms and alcohol consequences in college students. Addict Behav 35, 343-349.

81. Cruwys T, Platow MJ, Angullia SA, et al. (2012) Modeling of food intake is moderated by salient psychological group membership. Appetite 58, 754-757.

82. Tarrant M \& Butler K (2010) Effects of self-categorization on orientation towards health. BrJ Soc Psychol 50, 121-139.

83. Guendelman MD, Cheryan S \& Monin B (2011) Fitting in but getting fat: identity threat and dietary choice among U.S. immigrant groups. Psychol Sci 22, 959-967.

84. Schultz WP, Nolan JM, Cialdini RB, et al. (2007) The constructive, destructive, and reconstructive power of social norms. Psychol Sci 5, 429-434.

85. Steinberg L (2005) Cognitive and affective development in adolescence. Trends Cogn Sci 9, 69-74.

86. Mwamwenda TS (1995) Age differences in social desirability. Psychol Rep 76, 825-826.

87. Horne PJ, Tapper K, Lowe CF, et al. (2004) Increasing children's fruit and vegetable consumption: a peer-modelling and rewards-based intervention. Eur J Clin Nutr 58, 1640-1660.

88. Lowe CF, Horne PJ, Hardman CA, et al. (2006) Peermodelling and rewards-based intervention is effective in increasing fruit and vegetable consumption in children. Prev Med 43, 351-352.

89. Pachucki MA, Jacques PF \& Christakis NA (2011) Social network concordance in food choice among spouses, friends and siblings. Am J Public Health 101, 217-227.

90. Robinson E \& Higgs S (2012) Liking food less: social influence on food liking evaluations. PLOS ONE 7, e48858.

91. Hermans RC, Herman CP, Larsen JK, et al. (2010) Social modeling effects on snack intake among young men. The role of hunger. Appetite 54, 378-383.

92. Schachter S \& Gross LP (1968) Manipulated time and eating behavior. J Pers Soc Psychol 10, 98-106. 